\title{
矢车菊式-辣根过氧化物酶-过氧化氢新体系及其在 酶联免疫传感分析中的应用
}

\author{
龚福春*，李定中，杨荣，魏建科，曹忠，谭淑珍，谭亚非 \\ 长沙理工大学化学与生物工程学院, 长沙 410004 \\ * 通讯作者, E-mail: gfc139@yahoo.com.cn
}

收稿日期：2008-11-08; 接受日期：2008-12-09

摘要以一种天然色素矢车菊素-3-葡萄糖苷 (Cyanidin-3-glucose, CAG) 为辣根过 氧化物酶(HRP) 底物建立了矢车菊武-辣根过氧化物酶-过氧化氢新体系. CAG在 540 $\mathrm{nm}$ 处有一个吸收峰 $\left(A_{\mathrm{P} 1}\right)$, 在 $\mathrm{HRP}$ 催化下被 $\mathrm{H}_{2} \mathrm{O}_{2}$ 氧化, $\mathrm{A}_{\mathrm{P} 1}$ 降低; 同时, 其氧化产物在 $482 \mathrm{~nm}$ 处产生一个新吸收峰 $\left(A_{\mathrm{P} 2}\right)$, 并且反应体系中两峰值的比值 $R\left(A_{\mathrm{P} 2} / A_{\mathrm{P} 1}\right)$ 与 $\mathrm{HRP}$ 量 在一定浓度范围内呈线性相关. 根据此原理, 以猪口蹄疫病毒蛋白(food and mouth disease virus antigen, FMDVAg) 为分析模型, 建立了检测猪口蹄疫病毒的酶联免疫传 感分析新方法. 该方法利用伴刀豆蛋白(Conconvalina, ConA)对糖蛋白的识别特性固 定HRP-猪口蹄疫病毒抗体连接物(HRP-FMDVAb), 由ConA介导和磁性分离实现了 免疫反应特异性组分和非特异成分, 以及反应免疫磁性珠与没反应免疫磁性珠的分 离. 运用制备的传感体系测定猪口蹄疫病毒的线性范围为 $1.5 \times 10^{-8} \sim 2.7 \times 10^{-6} \mathrm{~g} / \mathrm{mL}$, 抗原检出限为 $3.1 \times 10^{-9} \mathrm{~g} / \mathrm{mL}$, 相对标准偏差为 $3.7 \%(n=11)$. 底物 CAG及其产物的水 溶性好, 对人体无毒副作用, 在临床上可代替传统HRP底物进行免疫检测.

关键词

矢车菊式

HRP 底物

酶联免疫传感

猪口蹄疫病毒
免疫分析是临床诊断、食品和药物分析以及环境 化学等领域中最常用的分析方法之一. 酶联免疫分 析将酶的催化放大作用和抗原抗体反应的专一性相 结合, 具有很高的选择性和灵敏度, 特别适合于低浓 度的样品和含有大量蛋白质、氨基酸、糖类等干扰物 的临床样品检测 $[1,2]$. 辣根过氧化物酶(HRP)是广泛应 用于酶联免疫分析(ELISA)中的一种标记酶 ${ }^{[3]}$, 鉴于 其优良特性, 已建立了各种光学酶联免疫分析 ${ }^{[4,3]}$ 和 电化学酶联免疫分析等体系 ${ }^{[6,7]}$. 酶免疫检测方法的 特征在很大程度上取决于所用的酶-底物系统. 传统 HRP底物主要有邻苯二胺 $(O P D) 、 3,3^{\prime}, 5,5^{\prime}$-四甲替联苯 胺(TMB)、5-氨基水杨酸(5-AS)、联大茴香胺(OD)、
邻联苯甲胺(OT)、3,3'-二氨基联苯胺(DAB)和 2,2'-连 胺基-2(3-乙基-并噻唑啉磺酸-6)铵盐(ABTS)等, 这些 底物存在如下不足: (1) OPD、TMB、5-AS、OD、 $\mathrm{OT}$ 及 DAB的水溶性差, 其水溶液在空气中不稳定, 需新鲜配制 ${ }^{[8]} ;(2)$ 多数酶催化产物水溶差, 不便于 比色定量测定, 例如组化法常用的HRP底物 3,3'-二氨 基联苯胺盐酸盐(DAB), 其反应物由于形成的氧化型 中间体迅速聚合, 形成不溶性的棕色吩溙衍生物 ${ }^{[9]}$, 只 能用于染色定性测定; (3) 有的底物对人体健康有害, 例如OPD可以使人体致癌 ${ }^{[10]}$ 等. 因此, 寻找更敏感、稳 定、安全无毒的HRP新底物对发展酶联免疫分析方法 具有重要意义. 
本课题组前期工作开发了几种性能优异的新底 物, 如白藜芦醇、 $2,3,5,4$ '-四羟基二苯乙烯-2- $O-\beta-D-$ 葡萄糖式和 4-差基苯乙烯基吡啶，并分别将其应用 于酶联免疫反应体系测定不同的抗原抗体. 本文建 立了矢车菊素-3-葡萄糖苷(CAG)-辣根过氧化物酶-过 氧化氢新体系. 对新体系的紫外-可见光谱特性进行 了研究, 发现 $\mathrm{CAG}$ 及其氧化产物分别在 $540 \mathrm{~nm}$ 和 $482 \mathrm{~nm}$ 处有一个紫外吸收峰, 并且两峰值的比值 $\mathrm{R}$ 与 HRP 在一定浓度范围内呈线性相关. 以猪口蹄疫 病毒抗原蛋白(Foot and mouth disease virus, FMDVAg) 为分析模型, CAG 为 HRP 底物, 建立了检测猪口蹄疫 病毒的酶联免疫传感分析新方法. 该方法利用伴刀 豆蛋白(Conconvalina, ConA)对糖蛋白的识别特性和 磁性分离同时实现了对 HRP-猪口蹄疫病毒抗体 (HRP-FMDVAb)固定, 以及免疫反应特异性组分和非 特异成分、反应免疫磁性珠与没反应免疫磁性珠分离. 该 CAG 及其氧化产物均有很好的水溶性, 储备液在 室温下稳定. 该体系可用于酶联免疫光度分析，具有 操作简便、灵敏度较高、试剂稳定等优点.

\section{1 实验部分}

\section{1 仪器与试剂}

样品的紫外-可见光谱测定在Shimadzu UV-1601 $P C$ 型紫外-可见分光光度计上进行. 矢车菊素-3-葡萄 糖苷 $(\mathrm{CAG}) 、 \beta-D$-甲基葡萄糖苷和伴刀豆蛋白从 Sigma公司获得; 邻苯二胺(OPD)、 $3,3^{\prime}, 5,5^{\prime}$-四甲替联 苯胺(TMB)、5-氨基水杨酸(5-AS)和 3,3'-二氨基联苯 胺(DAB)购于中国医药集团上海化学试剂公司; 甲基 三甲氧基硅烷(MTEOS)从武汉大学试剂厂购买; 聚 乙烯亚胺 (polyethyleneimine, PEI, $M_{n} \sim 400$ )购自东京 化成株式会社; 猪口蹄疫病毒抗原(FMDVAg)和猪口 蹄疫病毒抗体(FMDVAb)为中国农业部青岛动物检疫 研究所龚振华副研究员赠品; $\mathrm{Fe}_{3} \mathrm{O}_{4}$ 磁性纳米粒子 $(25$ $\mathrm{nm}$ ) 由长沙理工大学物理与电子学院提供. 辣根过 氧化物酶标记猪口蹄疫病毒抗体(HRP-FMDVAb)按 文献方法 ${ }^{[11]}$ 合成. 为本实验所用其他试剂均为国产 试剂.

$1 \mathrm{mmol} / \mathrm{L}$ 矢车菊式和 $0.01 \mathrm{~mol} / \mathrm{L} \mathrm{H}_{2} \mathrm{O}_{2}$ 储备液: 称 取 $4.85 \mathrm{mg}$ 矢车菊素-3-葡萄糖苷溶于 $100 \mathrm{~mL} \mathrm{pH} 6.8$ 的B-R缓冲液，即得底物储备液; 将 $0.28 \mathrm{~mL} 30 \%$
$\mathrm{H}_{2} \mathrm{O}_{2}$ 用 $\mathrm{pH} 6.8$ 的B- $\mathrm{R}$ 缓冲液定容至 $250 \mathrm{~mL}$ 制得 0.01 $\mathrm{mol} / \mathrm{L} \mathrm{H}_{2} \mathrm{O}_{2}$ 储备液.

\section{2 矢车菊式-辣根过氧化物酶-过氧化氢反应体 系测试}

于 $10 \mathrm{~mL}$ 的试管中加入 $1 \mathrm{~mL}$ 矢车菊式储备溶液, 用pH6.8 的B-R缓冲液稀释至刻度, 在 $35^{\circ} \mathrm{C}$ 下分别加 入 $0.2 \mathrm{~mL}$ 浓度为 $2.00 \times 10^{-3} \mathrm{~mol} / \mathrm{L} \mathrm{H}_{2} \mathrm{O}_{2}$ 和 $2.00 \times 10^{-3}$ $\mathrm{mol} / \mathrm{L} \mathrm{H}_{2} \mathrm{O}_{2}+1.00 \times 10^{-6} \mathrm{~mol} / \mathrm{L}$ HRP-FMDVAb, 反应 1 $\mathrm{min}$ 后, 用 $2 \mathrm{~mol} / \mathrm{L} \mathrm{H}_{2} \mathrm{SO}_{4}$ 终止反应. 在紫外-可见分光 光度仪上记录矢车菊式溶液的紫外可见吸收变化值. 以邻苯二胺 $(\mathrm{OPD}) 、 3,3^{\prime}, 5,5^{\prime}$-四甲替联苯胺(TMB)、5氨基水杨酸 (5-AS) 和 3,3'-二氨基联苯胺(DAB)作为对 照, 进行相同实验.

\section{3 抗体在PEI-Fe $\mathrm{F}_{3} \mathrm{O}_{4}$ 磁性微珠上的固定}

$\mathrm{PEI}-\mathrm{Fe}_{3} \mathrm{O}_{4}$ 聚合物磁性微珠的制备参照文献[12] 进行: 取 $60 \mathrm{~mL}$ 质量分数为 $2 \%$ 的PEI溶液于三颈烧瓶 中, 在 $\mathrm{N}_{2}$ 保护及快速搅拌下加入 $15 \mathrm{mg} \mathrm{Fe}_{3} \mathrm{O}_{4}$ 纳米粒 子及 $100 \mu \mathrm{L} 1.5 \mathrm{~N} \mathrm{FeCl}_{2}$, 搅拌混合, 加入 $2.5 \mathrm{~mL}$ 质量 分数为 $30 \%$ 的 $\mathrm{H}_{2} \mathrm{O}_{2}$, 同时进行紫外光照射, 反应 30 $\min$. 反应完成后, 磁性分离, 用三蒸水清洗, 即得 $\mathrm{PEI}$ 修饰的 $\mathrm{Fe}_{3} \mathrm{O}_{4}$ 磁性微珠, 颗粒大小为 $65 \mathrm{~nm}$ 左右(图 $1)$, 保存备用.

取PEI- $\mathrm{Fe}_{3} \mathrm{O}_{4}$ 磁性微珠 $50 \mathrm{mg}$ 于烧瓶中, 加入三蒸 水 $15 \mathrm{~mL}$ 分散, 然后加入 $3 \mathrm{~mL}$ 质量分数为 $2 \%$ 的戊二 醛和 $2 \mathrm{~mL}$ 质量分数为 $0.5 \%$ 的猪口蹄疫病毒抗体 (FMDVAb), 在 $28^{\circ} \mathrm{C}$ 恒温水浴条件下搅拌反应 $45 \mathrm{~min}$. 反应毕, 磁性分离, 用三蒸水清洗后, 将FMDVAb修 饰磁性纳米颗粒(FMDVAb-P)悬浮于 $20 \mathrm{~mL}$ pH6.8 的 B-R缓冲液中，保存备用.
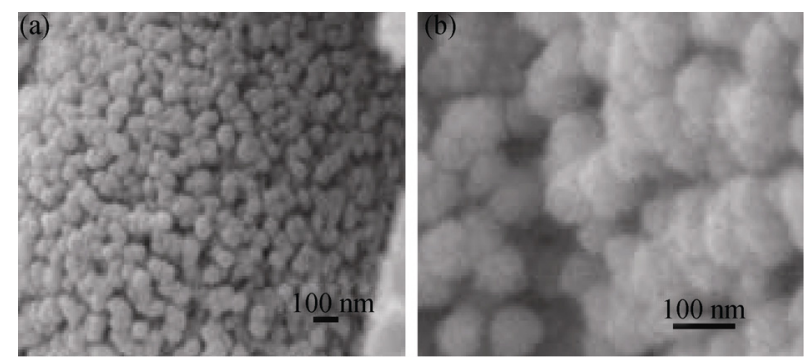

图 1 PEI-Fe $3_{3} \mathrm{O}_{4}$ 磁性微珠的扫描电子显微镜图 (a) 低倍; (b) 高倍 


\section{4 猪口蹄疫病毒抗原传感体系的制备}

按参考文献[13]利用溶胶-凝胶技术，以四甲氧 基硅 (TMOS) 和 $\gamma$-氨丙基甲基二甲氧基硅烷 (APMDMOS)为前驱体制备一种氨基化硅胶基质. 并 以戊二醛为交联剂，利用该氨基化硅胶为载体对 ConA进行交联固定化. 具体步骤: 一种含有 $6.5 \mathrm{~mL}$ 甲基三甲氧基硅烷、 $1.5 \mathrm{~mL} \gamma$-氨丙基甲基二甲氧基硅 烷、 $5.5 \mathrm{~mL}$ 水和 $2 \mathrm{~mL} 0.05 \mathrm{~mol} / \mathrm{L} \mathrm{HCl}$ 的混合溶液经 超声振荡器超声分散 15 min后, 室温放置水解 $36 \mathrm{~h}$, 获得约 $7.1 \mathrm{~g}$ 硅溶-胶凝(sol-gel)泥. 取 $5.0 \mathrm{~g}$ sol-gel泥 填充于一根内径为 $6 \mathrm{~mm}$ 的 $\mathrm{PVC}$ 管内, 制得一种含氨 基的硅胶支持棒，在进一步胶化或老化后 $\left(28^{\circ} \mathrm{C}, 24 \mathrm{~h}\right)$ 可用于ConA的固定.

将上述硅胶棒开口一端的表面沾水, 在 $\mathrm{Al}_{2} \mathrm{O}_{3}$ 砂 纸上打磨光亮后, 用 $\mathrm{pH} 6.8$ 的B-R缓冲液冲洗. 取 50 $\mu \mathrm{L} 5 \%$ 的戊二醛滴加在更新的表面，室温放置 $20 \mathrm{~min}$, 用蒸馏水洗掉未反应的戊二醛，然后将处理的硅胶 棒在浓度为 $1.5 \times 10^{-4} \mathrm{~mol} / \mathrm{L}$ 的伴刀豆蛋白溶液中培 育 $20 \mathrm{~min}$, 冲洗后，从而将伴刀豆蛋白固定在硅胶棒 上. 最后，将伴刀豆蛋白修饰的硅胶棒在 $5.5 \times 10^{-6}$
mol/L HRP 标记的猪口蹄疫病毒抗体(HRP-FMDVAb) 的溶液中 $37^{\circ} \mathrm{C}$ 培育 $45 \mathrm{~min}$, 用 $\mathrm{pH} 6.8$ 的 $\mathrm{B}-\mathrm{R}$ 缓冲液 冲洗后, 即获得猪口蹄疫病毒抗原传感体(CoA-HRPFMDVAb).

\section{5 酶联免疫传感分析程序}

酶联免疫传感测定程序如图 2 所示．首先将猪口 蹄疫病毒抗原传感体在含待测 FMDVAg 和 FMDVAb 修饰磁性纳米颗粒(FMDVAb-P)的混合溶液中 $37^{\circ} \mathrm{C}$ 培 育 $30 \mathrm{~min}$ 后，用 $\mathrm{pH} 6.8$ 的 B-R 缓冲液冲洗，除去非特 异性吸附的 FMDVAg 和 FMDVAb-P.接着, 将免疫反 应后的硅胶棒在 5\%的 $\beta$ - $D$-甲基葡萄糖苷溶液浸泡 15 min, 再用磁铁吸引脱附的HRP-FMDVAb-FMDVAgFMDVAb-P, 用pH6.8 的B-R缓冲液冲洗，保存备用。 最后, 进行酶反应和测定: 将磁铁吸附的 HRPFMDVAb-FMDVAg-FMDVAb-P固定于位于光路上的 流通反应池上，将包含 $6 \times 10^{-4} \mathrm{~mol} / \mathrm{L}$ 矢车菊式底物 溶液洜入流通反应池，测定 540 和 $482 \mathrm{~nm}$ 两处的吸光 度, 接着加入 $1.0 \times 10^{-4} \mathrm{~mol} / \mathrm{L} \mathrm{H}_{2} \mathrm{O}_{2}$ 并记录变化了的 吸光度, 计算两峰处吸光值的比值 $R$.

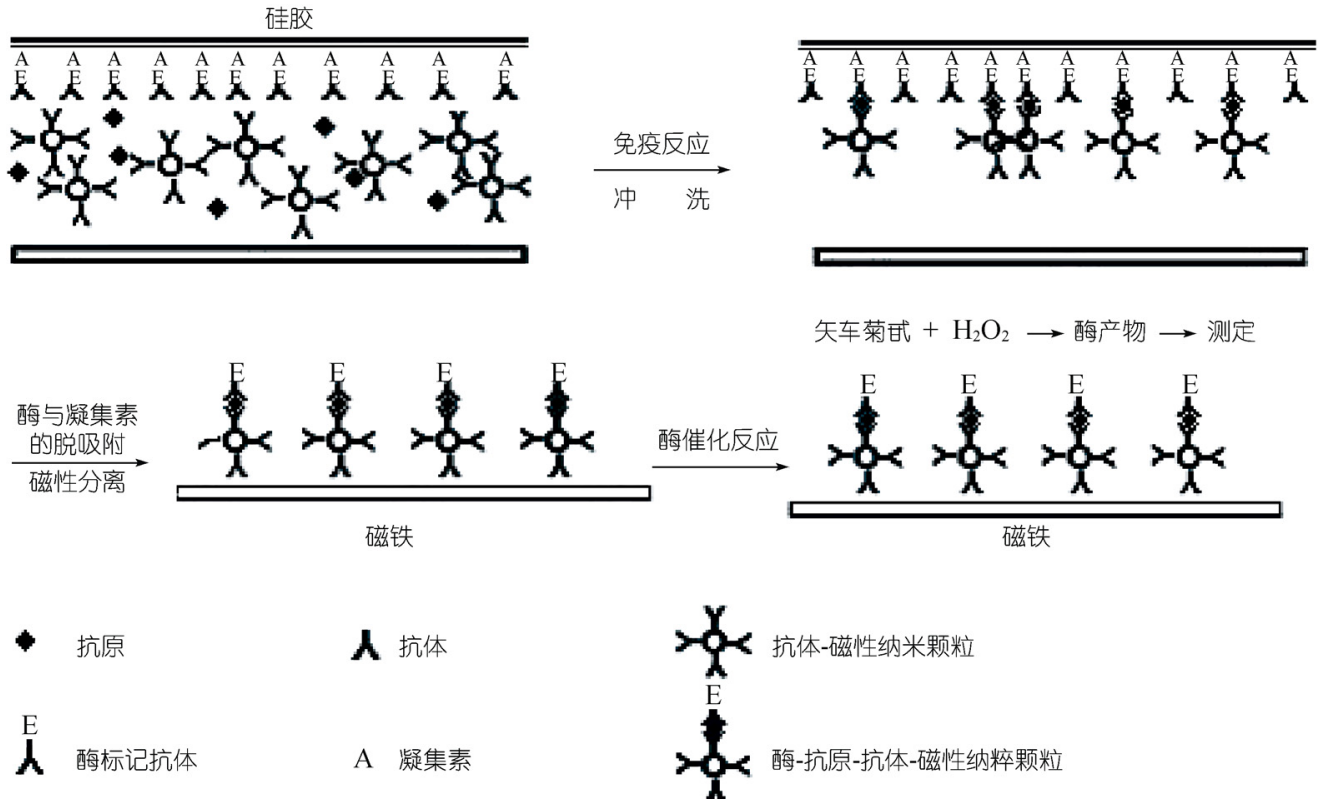

图 2 免疫分析程序 


\section{2 结果与讨论}

\section{1 矢车菊式-辣根过氧化物酶-过氧化氢反应体 系的特性}

对矢车菊式对 $\mathrm{H}_{2} \mathrm{O}_{2}$ 和HRP-FMDVAb $+\mathrm{H}_{2} \mathrm{O}_{2}$ 响应 性能进行了研究.表 1 是矢车菊式 $(\mathrm{CAG})$ 、邻苯二胺 (OPD)、3, 3',5, ' '-四甲替联苯胺(TMB)、5-氨基水杨 酸 (5-AS) 和 3, $3^{\prime}$-二氨基联苯胺 (DAB) 对 HRP$\mathrm{FMDVAb}+\mathrm{H}_{2} \mathrm{O}_{2}$ 及单独 $\mathrm{H}_{2} \mathrm{O}_{2}$ 反应的紫外吸光度数据, 从表中可知, $\mathrm{CAG}$ 对单独 $\mathrm{H}_{2} \mathrm{O}_{2}$ 的响应比 $\mathrm{OPD} 、 \mathrm{TMB}$ 和 $\mathrm{DAB}$ 小，与 5-AS相当; 但对 HRP-FMDVAb $+\mathrm{H}_{2} \mathrm{O}_{2}$ 的 响应值则与 $\mathrm{TMB}$ 和DAB相当, 比 5-AS的值要大.矢车 菊式溶液对单独 $\mathrm{H}_{2} \mathrm{O}_{2}$ 响应小, 可以较好地降低背景 信号，增加灵敏性; 对HRP-FMDVAb响应大，说明酶 对催化CAG氧化反应速率大,灵敏高.

表 1 加入 $\mathrm{H}_{2} \mathrm{O}_{2}$ 和 HRP-FMDVAb+ $\mathrm{H}_{2} \mathrm{O}_{2}$ 后底物矢车菊式 $(C A G) 、$ 邻苯二胺 $(O P D) 、 3,3^{\prime}, 5,5^{\prime}$ 四甲替联苯胺 $(T M B) 、$ 5-氨基水杨酸 (5-AS)和 3,3'-二氨基联苯胺(DAB)的紫外吸 收值变化率 $(\Delta A)$

\begin{tabular}{lcc}
\hline \multicolumn{1}{c}{$\mathrm{HRP}$ 底物 ${ }^{\mathrm{a})}$} & 加 $\mathrm{H}_{2} \mathrm{O}_{2}{ }^{\mathrm{b})}(\Delta A)$ & 加 $\mathrm{HRP} / \mathrm{H}_{2} \mathrm{O}_{2}{ }^{\mathrm{b})}(\Delta A)$ \\
\hline 矢车菊式 & 0.03 & 0.42 \\
邻苯二胺 & 0.12 & 0.43 \\
3,3',5,5'四甲替联苯胺 & 0.11 & 0.39 \\
5-氨基水杨酸 & 0.04 & 0.16 \\
$3,3^{\prime}$ 一二氨基联苯胺 & 0.12 & 0.44 \\
\hline
\end{tabular}

a) 底物浓度均为 $6 \times 10^{-4} \mathrm{~mol} / \mathrm{L} ;$ b) $\mathrm{H}_{2} \mathrm{O}_{2}$ 和 HRP-FMDVAb的 浓度分别为 $3.5 \times 10^{-3}$ 和 $1.2 \times 10^{-7} \mathrm{~mol} / \mathrm{L}$ ，反应时间为 $2 \mathrm{~min}$ ，用 2 $\mathrm{mol} / \mathrm{L} \mathrm{H}_{2} \mathrm{SO}_{4}$ 终止反应

对底物矢车菊式最佳反应浓度及体系的 $K_{\mathrm{m}}$ 进行 了研究.底物矢车菊式最佳反应浓度：加 $3 \mathrm{~mL} \mathrm{pH} 6.8$ 的B-R缓冲液于 $10 \mathrm{~mL}$ 的试管中, 再加 $50 \mu \mathrm{L} 2.5 \times 10^{-7}$ $\mathrm{mol} / \mathrm{L} \mathrm{HRP}$ 和 $50 \mu \mathrm{L} 1.00 \times 10^{-4} \mathrm{~mol} / \mathrm{L} \mathrm{H}_{2} \mathrm{O}_{2}$, 最后加不 同浓度的底物，定容到 $10 \mathrm{~mL}$ ，反应 $2 \mathrm{~min}$, 用 $2 \mathrm{~mol} / \mathrm{L}$ $\mathrm{H}_{2} \mathrm{SO}_{4}$ 终止反应, 测定吸光值. 由结果可知, 矢车菊式 浓度较低时，底物浓度与可见吸收强度呈线性关系， 当浓度大于 $6 \times 10^{-4} \mathrm{~mol} / \mathrm{L}$ 时紫外吸收强度值出现平台, 浓度达到 $6.5 \times 10^{-4} \mathrm{~mol} / \mathrm{L}$ 时吸光值最大.

利用初速度法测定了CAG-HRP- $\mathrm{H}_{2} \mathrm{O}_{2}$ 体系的 $K_{\mathrm{m}}$ 值. 反应条件同上, 取反应初速，作 $v-C_{\mathrm{CAG}}$ 图. 由图上 任取几个点, 求其倒数, 用双倒数法作图, 得一直线.
该体系线性拟合方程为 $1 / v=5.7 \times 10^{-13} \times 1 / C_{\mathrm{CAG}}+$ $3.5 \times 10^{-3}$; 变异系数 $r=0.994, V_{\max }=2.5 \times 10^{2} \mathrm{~mol} \cdot \mathrm{s}^{-1}$; 米氏常数 $K_{\mathrm{m}}=1.3 \times 10^{-4} \mathrm{~mol} / \mathrm{L}$. 由同样方法求得 $3,3^{\prime}, 5,5^{\prime}$ 四甲替联苯胺 $(\mathrm{TMB})$ 的 $V_{\text {max }}$ 为 $1.2 \times 10^{2} \mathrm{~mol} \cdot \mathrm{s}^{-1}$. 这说明CAG-HRP- $\mathrm{H}_{2} \mathrm{O}_{2}$ 体系比TMB-HRP- $\mathrm{H}_{2} \mathrm{O}_{2}$ 体系的 反应速率大.

\section{2 矢车菊式及其产物的紫外-可见光谱特性及免 疫分析原理}

对以 $\mathrm{CAG}$ 为 $\mathrm{HRP}$ 底物反应体系的紫外-可见光谱 进行了研究. 图 3 是矢车菊式及其HRP催化产物的可 见光谱, 由图可知, 底物在 $540 \mathrm{~nm}$ 处有个吸收峰 $\left(A_{\mathrm{P} 1}\right)$, $\mathrm{HRP}$ 催化 $\mathrm{H}_{2} \mathrm{O}_{2}$ 氧化矢车菊式, 生成的反应产物在 482 $\mathrm{nm}$ 处有一个新的最大吸收峰 $\left(A_{\mathrm{P} 2}\right)$, 并且随着 HRP浓 度增加, 吸收值 $\left(A_{\mathrm{P} 1}\right)$ 减少, 同时产物的吸收值 $\left(A \mathrm{p}_{2}\right)$ 增 加. 两吸收值之比值 $\left(A \mathrm{p}_{2} / A \mathrm{p}_{1}\right) R$ 与 $\mathrm{HRP}$ 的量在一定范 围内呈线性相关. 本酶联免疫分析方法通过测定 $R$ 值 来定量HRP，进而间接定量测定猪口蹄疫病毒抗原 (FMDVAg)浓度.

由于本方法采用两峰的比值来定量 FMDVAg 克 服了传统直接测定酶产物吸收的方法易受背景信号 干扰大的问题,提高了分析的灵敏度.

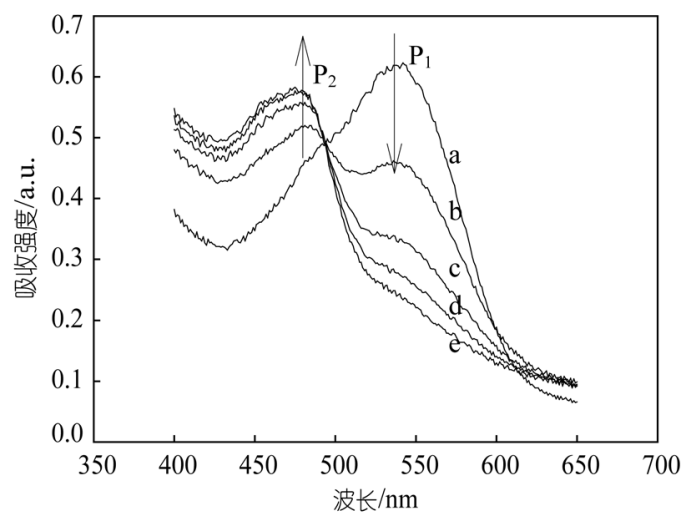

图 $3 \mathrm{CAG}$ 及其 HRP 催化产物的可见光谱 a. $6 \times 10^{-4} \mathrm{~mol} / \mathrm{LCAG}+3.5 \times 10^{-4} \mathrm{~mol} / \mathrm{L} \mathrm{H}_{2} \mathrm{O}_{2}$, 以及a 加入不同浓度的 HRP-FMDVAb: b. 1 ; c. 2 ; d. 4 ; e. $6\left(\times 10^{-8} \mathrm{~mol} / \mathrm{L}\right)$

\section{3 测定条件的选择}

用底物 $\mathrm{CAG}$ 溶液在 $540 \mathrm{~nm}$ 处的可见吸收强度表 示HRP-FMDVAb对CAG的催化活性. 根据吸收峰高 
低选择了 HRP-FMDVAb催化反应缓冲液及其 $\mathrm{pH}$. 对 B-R、HCl-Tris、 $\mathrm{Na}_{2} \mathrm{CO}_{3}-\mathrm{NaHCO}_{3} 、 \mathrm{NaOH}-\mathrm{H}_{3} \mathrm{BO}_{3}-\mathrm{KCl}$ 等几种缓冲液进行了比较, 结果表明: 在 $\mathrm{B}-\mathrm{R}$ 缓冲液 中, 响应较稳定.在 $\mathrm{pH} 6.8$ 时, $\mathrm{CAG}$ 的吸收峰型好, 响 应值最大.

对 $\mathrm{CAG}$ 和 $\mathrm{H}_{2} \mathrm{O}_{2}$ 的浓度对酶联免疫反应的影响进 行了测试. 结果显示, $\mathrm{CAG}$ 的浓度达到 $6 \times 10^{-4} \mathrm{~mol} / \mathrm{L}$ 时, 可以得到最大的吸收响应值; $\mathrm{H}_{2} \mathrm{O}_{2}$ 浓度为 $3.5 \times 10^{-4}$ $\mathrm{mol} / \mathrm{L}$ 时, 峰高变化最大, 超过此浓度后, 随着 $\mathrm{H}_{2} \mathrm{O}_{2}$ 浓度的增加, 峰高增加值变小, 这一现象体现了 $\mathrm{H}_{2} \mathrm{O}_{2}$ 既是HRP的底物又是其抑制剂的双重性.

鉴于免疫组分一般在动物体温下活力较好的特性, 所以，传感体在免疫反应液的培育温度选择为 $37^{\circ} \mathrm{C}$. 考察了 HRP-FMDVAb催化CAG 与 $\mathrm{H}_{2} \mathrm{O}_{2}$ 反应的最适温 度. 实验结果表明: 反应温度在 $30 \sim 40^{\circ} \mathrm{C}$ 范围内, 吸收 响应较大. 本实验选择 $37^{\circ} \mathrm{C}$ 作为酶催化反应温度.

\section{4 线性范围、检测限及 FMDVAg 的测定}

按实验部分所述方法, 以 $\mathrm{CAG}$ 为 HRP-FMDVAb 底物，在所选择的最优条件下，测得 $\mathrm{R}$ 响应值与猪口 蹄疫病毒蛋白抗原浓度在 $1.5 \times 10^{-8} \sim 2.7 \times 10^{-6} \mathrm{~g} / \mathrm{mL}$ 范围内呈线性关系, 其回归方程为: $R=0.031+42.8 C$ $(\mathrm{g} / \mathrm{mL}), r^{2}=0.9983$. 对浓度为 $3.0 \times 10^{-7} \mathrm{~g} / \mathrm{mL}$ 的猪口 蹄疫病毒蛋白抗原连续进行 11 次平行测定, 得相对
标准偏差为 $3.7 \%$, 抗原检出限为 $3.1 \times 10^{-9} \mathrm{~g} / \mathrm{mL}$. 结 果表明: 本体系的灵敏度稍优于诊断试剂盒所用 $\mathrm{TMB}$ 底物 $\left(5.4 \times 10^{-9} \mathrm{~g} / \mathrm{mL}\right)$, 而且所用底物矢车菊素3-葡萄糖苷比TMB更稳定, 无需避光保存.

\section{5 样品回收测试}

按照上述实验方法，对猪口蹄疫病毒抗原蛋白 进行了回收率实验, 所得结果见表 2. 由表中数据可 以看出本方法稳定性和灵敏性均较好.

\section{6 机理讨论}

从花色苷的结构看, 由于共轭效应, 氧上的不成 对电子并不固定于氧原子, 而是靠近苯环, 从而削弱 氢氧键，使羟基上氢原子活性提高，易于脱去而成为 氢供体, 在植物体内是一种天然的抗氧化剂 ${ }^{[14,15]}$. 矢 车菊素-3-葡萄糖苷是一种多酚类物质，具有邻位的 羟基且数目较多.一方面有多次提供氢原子的能力, 另一方面其邻位羟基苯衍生物生成的苯氧基自由基 可在分子内部生成氢键而得到稳定，还可以通过电 子转移, 形成稳定的化合物, 从而提高其活性. 在矢 车菊式-辣根过氧化物酶-过氧化氢新体系中, 矢车菊 素-3-葡萄糖苷作为供氢体参与反应。由实验结果和 相关工作可推测, 矢车菊素-3-葡萄糖苷在HRP催化 下被 $\mathrm{H}_{2} \mathrm{O}_{2}$ 氧化的机理如图 4:

表 2 样品回收数据

\begin{tabular}{ccccc}
\hline 样品 & 起始浓度 $/ \mathrm{mg} \cdot \mathrm{L}^{-1}$ & 加入量 $/ \mathrm{mg} \cdot \mathrm{L}^{-1}$ & 回收量 $/ \mathrm{mg} \cdot \mathrm{L}^{-1}$ & 回收率 $(n=4) \%$ \\
\hline 标准 & 10.00 & 1.50 & 1.48 & 98.7 \\
FMDVAg & & 2.20 & 2.15 & 97.7 \\
血清 & 0 & 1.70 & 1.67 & 98.2 \\
& & 4.00 & 4.05 & 101.3 \\
\hline
\end{tabular}<smiles>CCOc1cc2c(O)cc(O)cc2c(Cl)c1C(=O)O</smiles>

图 4 HRP 催化矢车菊素-3-葡萄糖苷氧化反应机理 


\section{3 结论}

矢车菊素-3-葡萄糖苷及其产物水溶性好, 在空 气中稳定，对光温度不敏感，而对辣根过氧化物酶敏 感，且无毒副作用，是一种优良的 HRP 底物.将该新 HRP 底物运用于酶联免疫传感体系测定 FMDVAg, 结果表明: 基于新底物的酶联免疫分析方法灵敏较
高、检测下限低、稳定性好. 以伴刀豆蛋白为介体能 较好固定 HRP-FMDVAb 连接物, 将伴刀豆蛋白对糖 蛋白的吸附-解脱特性与磁性纳米粒子的磁性结合可 以较好地解决酶联免疫传感体系免疫反应复合物-磁 性纳米颗粒与没反应磁性纳米颗粒的分离问题, 为 提高灵敏度开辟了一条有效途径.

致谢本工作得到湖南省科技计划项目(批准号：2008SK3052)和湖南省教育厅科研项目(批准号：08B004, 07A006)资助.

\section{参考文献}

1 Meyer J, Karst U. Enzyme-linked immunosorbent assays based on peroxidase labels and enzyme-amplified lanthanide luminescence detection. Analyst, 2001, 126(24): 175-178 [DOI]

2 Jörg M, Uwe K, Enzyme-linked immunosorbent assay based on peroxidase labels and enzyme-amplified lanthanide luminescence detection. Analyst, 2000, 126(1): 175-178

3 Mohanty J G, Jaffe J S, Schulman E S, Raible D G. A highly sensitive fluorescent microassay of $\mathrm{H}_{2} \mathrm{O}_{2}$ release from activated human leukocytes using a dihydroxyphenoxazinederivative. J Immunol Meth, 1997, 202(6): 133-41[DOI]

4 Gong F C, Tang L F, Shen G L, Yu R Q. Fluorometric enzyme immunosensing system based on a renewable immunoreaction platform for the detection of Schistosoma japonicum antibody. Talanta, 2004, 62(4): 735-740[DOI]

5 Plowman T E, Reichert W M, Peters C R, Wang H K, Christensen D A, Herron J N. Femtomolar sensitivity using a channel-etched thin film waveguide fluoroimmunosensor. Biosens Bioelectron, 1996, 11(1-2): 149-160[DOI]

6 李建国, 刘颖, 鞠熀先. 季胺-辣根过氧化物酶-过氧化氢显色新体系及其在酶活性检测中的应用. 化学学报, 2007, 65(15): 1499-1503

7 Limoges B, Degrand C, Brossier P. Homogeneous electrochemical immunoassay using a persulfonated ionomer-modified electrode as a detector for a cationic labeled hapten. Anal Chem, 1993, 65: 1054-1060[DOI]

8 孙伟, 焦奎, 张书圣. 伏安酶联免疫分析中一类新的 HRP 底物体系的初步研究. 青岛化工学院学报(自然科学版), 2000, 21(4): $377-280$

9 Ghindilis A L, Krishnan R, Atanasov P, Wilkins E. Flow-through amperometric immunosensor: Fast 'sandwich' scheme immunoassay. Biosens Bioelectron, 1997, 12(5): 415-423 [DOI]

10 Alexander P W, Maltra C. Enzyme-linked immunoassay of human immunoglobulin G with the fluoride ion selective electrode. Anal Chem, 1982, 54: 68-71 $\underline{[\mathrm{DOI}]}$

11 Gong F C, Zhou Z J, Shen G L, Yu R Q. Schistosoma japonicum antibody assay by immunosensing with fluorescence detection using 3,3',5,5'-tetramethylbenzidine as substrate. Talanta, 2002, 58(4): 611-618[DOI]

12 刘洪玲, 李军. 肿瘤靶向 PEI 包覆磁性纳米凝胶的光化学制备及表征. 高等学校化学学报, 2008, 29(8): 1703-1706

13 钱军民, 李旭祥, 锁爱莉. 氨基化硅胶固定化葡萄糖氧化酶的研究. 生物化学与生物物理进展, 2002, 29(3): 13-17

14 初玉侠, 张惠祥, 金春光. 牵牛花色素的提取及其性质的研究. 食品科学, 1991, 5: 7-12

15 Florian C S, Angela S S, Reinhold C, Balz F, Ronald E W. Color and antioxidant properties of cyanidin-based anthocyanin pigments. J Agric Food Chem, 2002, 50(21): 6172-6181 [DOI] 